\title{
Hyperglycaemia in early pregnancy: the Treatment of Booking Gestational diabetes Mellitus (TOBOGM) study. A randomised controlled trial
}

David Simmons ${ }^{1}$, William M Hague ${ }^{2,3}$, Helena J Teede ${ }^{4}$, N Wah Cheung ${ }^{5}$, Emily J Hibbert ${ }^{6}$, Christopher J Nolan ${ }^{7,8}$, Michael J Peek ${ }^{8}$, Federico Girosi ${ }^{1}$, Christopher T Cowell ${ }^{5}$, Vincent W-M Wong ${ }^{9}$, Jeff R Flack ${ }^{10}$, Mark McLean ${ }^{11}$, Raiyomand Dalal ${ }^{12}$, Annette Robertson ${ }^{12}$, Rohit Rajagopal ${ }^{12}$

dentifying and managing women with gestational diabetes mellitus (GDM) from 24-28 weeks' gestation is now standard care in Australia and overseas; this practice is largely based on evidence from two randomised controlled trials (RCTs).$^{1-3}$ The World Health Organization and the International Association of Diabetes and Pregnancy Study Groups (IADPSG) also recommend screening for overt diabetes in pregnancy (ODIP) at the first antenatal booking appointment (usually by 14 weeks' gestation) $)^{4,5}$ to identify undiagnosed pre-gestational diabetes. When this assessment includes an oral glucose tolerance test (OGTT), the GDM diagnostic criteria applied are those for women who are 24-28 weeks pregnant. ${ }^{6}$ This early diagnosis, termed "booking GDM", accounts for $40-66 \%$ of GDM diagnoses, ${ }^{7-9}$ and can be associated with adverse pregnancy outcomes. ${ }^{7,8}$

However, there is no conclusive evidence that diagnosing and treating booking GDM is beneficial for mothers or their babies. Several studies ${ }^{10-12}$ have delivered results which suggest that overtreating the mother can lead to small for gestational age babies and possibly to genetic programming causing future metabolic disease. ${ }^{13}$ As fasting plasma glucose levels are elevated during the first trimester of pregnancy, ${ }^{14}$ applying the GDM diagnostic criteria for 24-28 weeks' gestation before 20 weeks' gestation may be inappropriate. In a large Chinese study, only $37 \%$ of women who had fasting glucose levels of $5.1-5.6 \mathrm{mmol} / \mathrm{L}$ at their booking visit but were not subsequently treated had GDM at 24-28 weeks' gestation. ${ }^{15}$ The IADPSG has now advised against formally diagnosing booking GDM according to the $24-28$ weeks criteria, ${ }^{16}$ but have not provided an alternative.

\section{Research question}

We will investigate whether treating pregnant women diagnosed before 20 weeks' gestation with hyperglycaemia according to the Australasian Diabetes in Pregnancy Society 2014 GDM criteria for women who are $24-28$ weeks pregnant ${ }^{3}$ improves obstetric outcomes.

\section{Methods}

\section{Study design}

The study design is summarised in Box 1. The Treatment Of Booking Gestational diabetes Mellitus (TOBOGM) study is a

\section{Abstract}

Background: Gestational diabetes mellitus (GDM) causes adverse pregnancy outcomes that can be averted by treatment from 24-28 weeks' gestation. Assessing and treating women for overt diabetes in pregnancy (ODIP) at the first antenatal clinic booking is now recommended in international guidelines. As a consequence, women with milder hyperglycaemia are being diagnosed and treated for early GDM, but randomised controlled trial (RCTs) assessing the benefits and harms of such treatment have not been undertaken. The Treatment of Booking Gestational diabetes Mellitus (TOBOGM) study is a multi-centre RCT examining whether diagnosing and treating GDM diagnosed at booking improves pregnancy outcomes.

Methods and analysis: 4000 adult pregnant women (< 20 weeks' gestation) at risk of ODIP will be recruited from 12 hospital antenatal booking clinics and referred for an oral glucose tolerance test (OGTT). 800 women with hyperglycaemia (ie, booking GDM) according to the 2014 Australasian Diabetes-in-Pregnancy Society criteria for pregnant women at 24-28 weeks' gestation will be randomised to immediate treatment for GDM (intervention) or to no treatment (control), pending the results of a second OGTT at 24-28 weeks' gestation. Antenatal and GDM care will otherwise follow local guidelines. Randomisation will be stratified by site and OGTT glycaemic risk strata. The primary pregnancy outcome is a composite of respiratory distress, phototherapy, birth trauma, birth before 37 weeks' gestation, stillbirth or death, shoulder dystocia, and birthweight $\geq 4.5 \mathrm{~kg}$. The primary neonatal outcome is neonatal lean body mass. The primary maternal outcome is pre-eclampsia.

Ethics approval: South Western Sydney Local Health District Research and Ethics Office (reference, 15/LPOOL/551).

Dissemination of results: Peer-reviewed publications, scientific meetings, collaboration with research groups undertaking comparable studies, discussions with guideline groups and policy makers.

Trial registration: Australian New Zealand Clinical Trials Registry, ACTRN12616000924459.

multi-centre RCT in which women with booking GDM are randomised to either early treatment (open label intervention group) or no treatment (double-blinded control group). A parallel cohort of women with normal OGTT results serve as decoys. Control and decoy participants undergo a second OGTT at

'Western Sydney University, Sydney, NSW. ${ }^{2}$ Robinson Research Institute, University of Adelaide, Adelaide, SA. ${ }^{3}$ Women's and Children's Hospital, Adelaide, SA. ${ }^{4}$ Monash

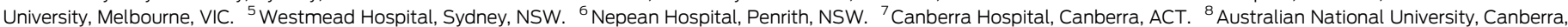

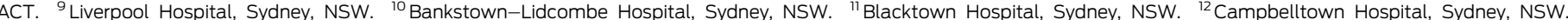
\. Da.simmons@westernsydney.edu.au • doi: 10.5694/mja17.01129 • Published online 28/05/2018 


\section{The Treatment of Booking Gestational diabetes Mellitus (TOBOGM)} study design

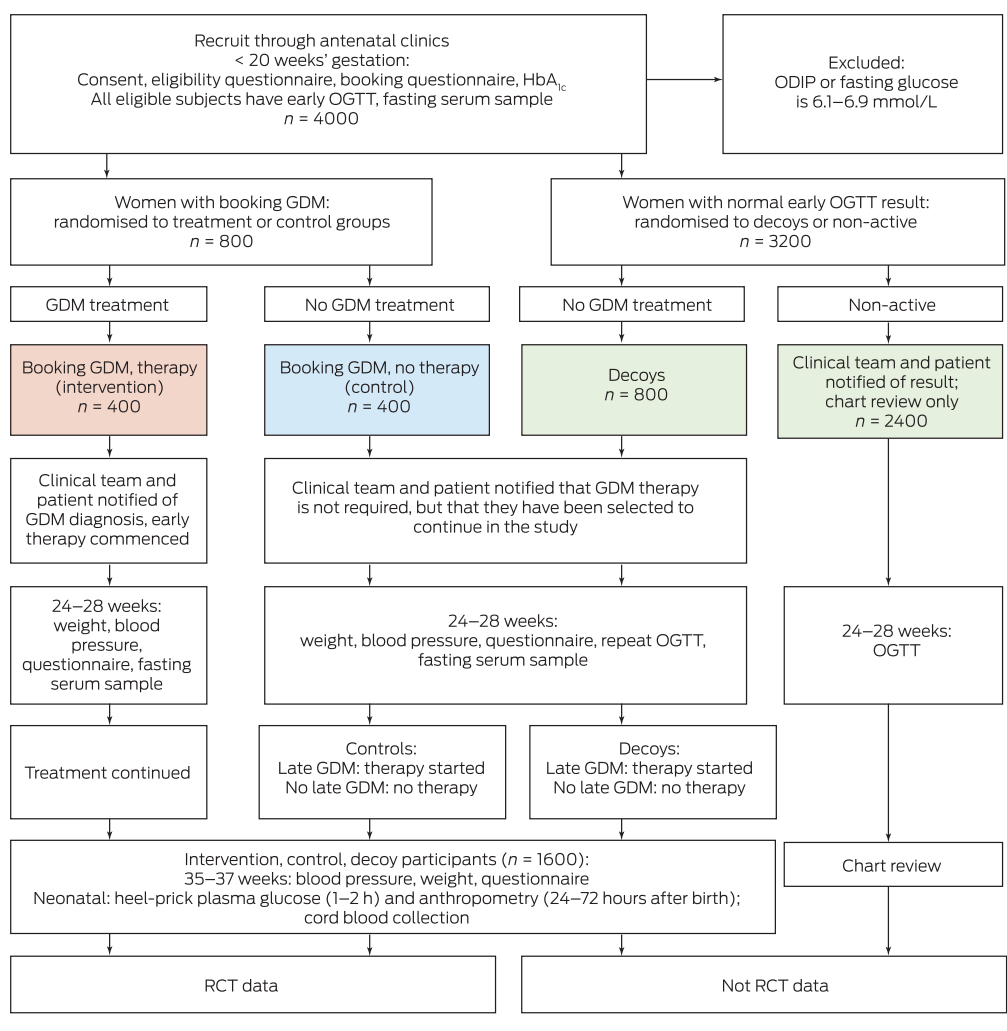

$\mathrm{GDM}=$ gestational diabetes mellitus; $\mathrm{HbA}_{1 \mathrm{c}}=$ glycated haemoglobin; ODIP = overt diabetes in pregnancy; OGTT $=$ oral glucose tolerance test; $\mathrm{RCT}=$ randomised control trial. $\bullet$
24-28 weeks' gestation; if this leads to a diagnosis of GDM, treatment will be started.

\section{Aims of the study}

- To define the prevalence and natural history of booking GDM.

- To determine whether treating booking GDM reduces the incidence of perinatal and maternal sequelae of maternal hyperglycaemia without increasing the risk of fetal undernutrition.

- To evaluate the cost-effectiveness of treating booking GDM.

\section{Study timetable and sites}

- Commence recruitment: May 2017.

- Complete recruitment: December 2018.

- Complete data collection: June 2019.

The participating sites will be Campbelltown Hospital (lead), Bankstown-Lidcombe Hospital, Liverpool Hospital, Nepean Hospital, Westmead Hospital, Blacktown Hospital (New South Wales), Canberra Hospital (Australian Capital Territory), Monash Health (Victoria), Women's and Children's Hospital, Flinders Medical Centre, Lyell McEwin Hospital (South Australia), and the Medical University of Vienna (Austria).

\section{Participant recruitment, randomisation and blinding}

Adult women ( $\geq 18$ years of age) attending hospital antenatal booking clinics at the participating sites are eligible for inclusion if they have a singleton pregnancy of $4-19^{+6}$ weeks' gestation, have a risk factor for ODIP, ${ }^{3}$ and provide signed informed consent. Women are excluded if they have pre-existing diabetes or other major active medical disorders.

Consecutive, consenting eligible women will be referred for an OGTT, to be performed as soon as practical and before 20 weeks' gestation. The results will be forwarded to the central study coordinating team (all hospital staff will be blinded to the OGTT values to prevent management bias) and double entered into a bespoke electronic randomisation program (Techtonic) applying block randomisation. Study sites will be electronically advised of the action required according to the blinded OGTT result and randomisation:

- Women with ODIP or a fasting glucose level of 6.1-6.9 mmol/L (who, by consensus, are considered to be at particularly high risk of diabetes) will be excluded from the trial, their results unblinded, and they will be referred for immediate treatment.

- Women with normal glucose tolerance according to the GDM criteria (fasting glucose level $<5.1 \mathrm{mmol} / \mathrm{L}$, one-hour glucose level $<10.0$ $\mathrm{mmol} / \mathrm{L}$, and 2-hour glucose level $<8.5 \mathrm{mmol} / \mathrm{L}$ ) will be advised that they do not require referral for GDM care. Of these women, 800 will be randomly selected to undergo all subsequent procedures (decoys), the others (about 2400) will receive usual antenatal care and have their records reviewed postnatally, but will not be asked to complete further questionnaires (non-active).

- Women fulfilling booking GDM criteria (fasting glucose level $\geq 5.1 \mathrm{mmol} / \mathrm{L}$, one-hour glucose level $\geq 10.0 \mathrm{mmol} / \mathrm{L}$, or 2-hour glucose level $\geq 8.5 \mathrm{mmol} / \mathrm{L}$ ) will be randomised to the intervention (referred for early GDM treatment) or control groups (no treatment, but to undergo OGTT re-testing and other assessment at 24-28 weeks). Randomisation will be stratified by site and by two glucose level strata, higher (HRV) and lower risk glucose values (LRV) (Box 2). The strata correspond to 1.75(LRV) and 2-fold risks (HRV) of adverse pregnancy outcomes according to OGTT results discussed by the IADPSG.

Women not randomised to the intervention (ie, control, decoy, and non-active participants) will undergo a second OGTT at 24-28 weeks; women diagnosed with GDM according to the 2014 Australasian Diabetes in Pregnancy Society criteria ${ }^{3}$ will be referred for immediate GDM management. Clinic and study staff will be blinded to the specific OGTT results of controls and decoys unless the fasting glucose level is at least $6.1 \mathrm{mmol} / \mathrm{L}$ or the 2-hour glucose level is at least $11.1 \mathrm{mmol} / \mathrm{L}$.

Obstetric management will follow usual practice. Should there be indications suggesting hyperglycaemia (eg, glycosuria, polyhydramnios), further open assessment of glucose tolerance will be permitted at the discretion of the attending clinician. Consensus GDM management guidelines are summarised in Box 3, including glycaemic targets that reflect those applied in earlier RCTs following the diagnosis of GDM at 24-28 weeks. ${ }^{1,2}$ Management will include education about GDM, dietary advice from a qualified dietitian, and instruction in self-monitoring of capillary blood glucose with the Accu-Chek Guide (Roche). Blood glucose data will be downloaded and clinical GDM reviews undertaken. Insulin or metformin therapy will be instituted according to local practice for women unable to maintain glucose level targets. All management changes will be recorded. 
2 Categories of dysglycaemia by oral glucose tolerance test (OGTT) result

\begin{tabular}{lccc} 
& \multicolumn{3}{c}{ OGTT value (mmol/L) } \\
\cline { 2 - 4 } Category & Fasting & One-hour & 2-hour \\
\hline Overt diabetes in pregnancy & $\geq 7.0$ & & $\geq 11.1$ \\
High fasting glucose & $6.1-6.9$ & & \\
Higher risk values & $5.3-6.0$ & $\geq 10.6$ & $9.0-11.0$ \\
Lower risk values & $5.1-5.2$ & $10.0-10.5$ & $8.5-8.9$
\end{tabular}

\section{Collection of maternal data}

Baseline demographic data, medical, obstetric, family, paternal and lifestyle history, and quality of life information will be collected with standard questionnaires. In addition to the OGTTs discussed above, blood for glycated haemoglobin $\left(\mathrm{HbA}_{1 \mathrm{c}}\right)$ measurement and a fasting serum sample for further assessment (including insulin, leptin, adiponectin, lipid levels) will be collected at the start of the first OGTT and stored. At 24-28 and 35-37 weeks' gestation, all participants except non-active women will complete a questionnaire on lifestyle, health service use, quality of life, and out-of-pocket health expenses. Pre-pregnancy weight, where known, will be recorded at the initial visit. Height and weight will be measured at the initial booking clinic visit, and used to calculate their body mass index (BMI; higher BMI is a risk factor for ODIP). Maternal weight and blood pressure will be recorded at each study time point. All women fulfilling criteria for GDM at booking (or subsequently), whether managed as GDM or not, will be asked to undertake an OGTT 6-12 weeks after giving birth. A postnatal questionnaire on breast feeding and quality of life will be completed by all women other than non-active participants (Box 4, Box 5).

\section{Collection of neonatal data and cord blood sampling}

Venous umbilical cord blood will be collected in EDTA tubes. Glucose will be measured in a neonatal heel-prick blood sample 1-2 hours after birth. Neonatal anthropometry (length; skin calliper measurements at four sites, including flank, upper arm circumference) will be assessed within 72 hours of birth. Other relevant data will be extracted from case notes and statutory data repositories.

\section{Blood sample processing and storage}

Blood samples for the OGTT will be collected according to American Diabetes Association recommendations. ${ }^{18}$ Maternal fasting blood collected for serum storage and cord blood will be separated as soon as possible (preferably within 30 minutes), initially stored at $-20^{\circ} \mathrm{C}$, and transferred within 48 hours for storage at $-80^{\circ} \mathrm{C}$.

\section{Primary and secondary outcomes}

The primary pregnancy outcome will be a composite of respiratory distress (defined as needing at least 4 hours' respiratory support with supplemental oxygen, continuous positive airway pressure, or intermittent positive pressure ventilation in the 24 hours after delivery), need for phototherapy, birth trauma (according to IADPSG criteria ${ }^{19}$ ), birth before 37 weeks' gestation, stillbirth or death, shoulder dystocia (vaginal cephalic delivery that requires additional obstetric manoeuvres to deliver the fetus after the head has delivered and gentle traction has failed), and birthweight $\geq 4.5 \mathrm{~kg}$.
3 Management of gestational diabetes in the Treatment of Booking Gestational diabetes Mellitus (TOBOGM) study

- Education: Group diabetes educator and dietitian review for all participants.

- Diet: Local guidelines, according to Dietitians Association of Australia usual practice.'

- Physical activity: Local practice based on Australasian Diabetes-inPregnancy Society guidelines. ${ }^{3}$

- Blood glucose level self-monitoring: Minimum four times/day (before breakfast, 2 hours after breakfast, lunch, dinner).

- Glycaemic targets (thresholds for intensification of therapy): $5.3 \mathrm{mmol} / \mathrm{L}$ (fasting), $7 \mathrm{mmol} / \mathrm{L}$ (2 hours), or two unexplained high blood glucose values within 7 days.

- Treatment intensification: Basal and/or rapid acting insulin therapy, tailored to the woman. Sites will have the choice to use metformin, but local policy should not change during the trial except in exceptional circumstances.

- Metformin treatment: Metformin will be used according to this standard approach:

- Start with standard metformin 1-3 $1500 \mathrm{mg} /$ day, depending on glucose profile, increasing to maximum $1-3 \times 1000 \mathrm{mg} /$ day.

- Long-acting (XR) metformin may be considered if standard metformin is not tolerated and insulin is refused.

- Insulin may be added to metformin treatment if control is not achieved with metformin alone.

The primary maternal outcome will be the incidence of pre-eclampsia (excluding women with chronic hypertension). The incidence of eclampsia and gestational hypertension will also be recorded. ${ }^{20}$

The primary neonatal outcome will be the neonatal lean body mass calculated with the Catalano equation; ${ }^{12}$ that is, birthweight minus fat mass, where fat mass $(\mathrm{kg})=0.39055 \times$ birthweight $(\mathrm{kg})+$ $0.0453 \times$ flank skinfold $(\mathrm{mm})-0.03237 \times$ length $(\mathrm{cm})+0.54657$.

The secondary outcomes will be birthweight, neonatal fat mass (Catalano equation), ${ }^{12}$ ethnic background-adjusted customised centile for birthweight (www.gestation.net), mean upper arm circumference, 1-2 hour neonatal heel-prick glucose level $\leq 2.2 \mathrm{mmol} / \mathrm{L}$, severe neonatal hypoglycaemia (any heelprick blood glucose level $<1.6 \mathrm{mmol} / \mathrm{L}$ ), neonatal intensive care unit bed-days, sum of neonatal skin calliper measurements, total maternal gestational weight gain, caesarean delivery, induction of labour, maternal hypoglycaemia (number of glucose measurements $<3.0 \mathrm{mmol} / \mathrm{L}$ ), perineal trauma, breast feeding, and quality of life.

\section{Statistical analyses}

Continuous outcomes will be analysed by analysis of variance (ANOVA). Non-normally distributed data will be analysed either after log transformation or with non-parametric methods. Linear regression analysis will account for potential confounders. Dichotomous outcomes (eg, primary maternal outcomes) will be analysed by logistic regression. Tukey-Kramer or Fisher-Hayter tests will be used for post hoc comparisons. Outcomes with multiple measurements at pre-defined time points (eg, maternal weight gain) will be analysed in linear mixed models using generalised estimating equations (GEE) for continuous outcomes. For the analysis of binary outcomes, logistic random intercept/GEE models will be applied.

Multiplicity. All analyses will be adjusted for clustering by site. When deemed appropriate, a method such as the TukeyCiminera-Heyse procedure - adjusted $P=1-(1-P)^{\sqrt{k}}$, where $k=$ number of comparisons - will be used to adjust for multiplicity. 
4 Schedule for participants in the Treatment of Booking Gestational diabetes Mellitus (TOBOGM) randomised controlled trial (intervention and control groups) and for decoys

\begin{tabular}{|c|c|c|c|c|c|c|}
\hline Data collection & Screening/booking & Prenatal & Prenatal & Birth & Post-birth & Postnatal \\
\hline Visit window & $\begin{array}{l}\text { Day of recruitment } \\
\quad(<20 \text { weeks })\end{array}$ & $\begin{array}{l}24-28 \\
\text { weeks }\end{array}$ & $\begin{array}{l}35-37 \\
\text { weeks }\end{array}$ & $\begin{array}{c}\text { At } \\
\text { birth }\end{array}$ & $\begin{array}{l}\text { Before discharge } \\
(24-72 \mathrm{~h})\end{array}$ & $\begin{array}{c}6 \\
\text { weeks }\end{array}$ \\
\hline \multicolumn{7}{|l|}{ Maternal procedures and measurements } \\
\hline Demographic data & $x$ & & & & & \\
\hline $\begin{array}{l}\text { Medical/obstetric history (including GDM risk } \\
\text { factors)* }\end{array}$ & $x$ & & & & & \\
\hline Health/quality of life questionnaire & $x$ & $x$ & $x$ & & & $x$ \\
\hline Weight & $x$ & $x$ & $x$ & & & \\
\hline Height & $x$ & & & & & \\
\hline Blood pressure & $x$ & $x$ & $x$ & $x$ & & \\
\hline OGTT booked & $x$ & $x^{\dagger}$ & & & & \\
\hline $\mathrm{HbA}_{1 \mathrm{c}}$ level & $x$ & $x^{\ddagger}$ & & & & \\
\hline Serum stored & $x$ & & & & & \\
\hline Record of hospitalisations & & & & & $x$ & \\
\hline Glucometer data download (if treated) & & $X^{\ddagger}$ & $X^{\ddagger}$ & & $x$ & \\
\hline Details of last ultrasound prior to birth & & & & & $x$ & \\
\hline GDM/birth information & & & & & $x$ & \\
\hline Postnatal OGTT (GDM women only) & & & & & & $x$ \\
\hline Adverse events & $x$ & $x$ & $x$ & $x$ & $x$ & $x$ \\
\hline Length of stay (birth and postnatal) & & & & & $x$ & \\
\hline \multicolumn{7}{|l|}{ Neonatal procedures and measurements } \\
\hline Neonatal information & & & & & $x$ & \\
\hline Neonatal outcomes & & & & & $x$ & \\
\hline Cord blood & & & & $x$ & & \\
\hline Heel-prick blood glucose (1-2 h) & & & & $x$ & & \\
\hline Anthropometric measurements ( $24-72 \mathrm{~h}$ ) & & & & & $x$ & \\
\hline Information about feeding & & & & & $x$ & $x$ \\
\hline NICU admission and length of stay & & & & & $x$ & $x$ \\
\hline
\end{tabular}

GDM = gestational diabetes mellitus; $\mathrm{HbA}_{\mathrm{lc}}=$ glycated haemoglobin; NICU = neonatal intensive care unit; OGTT = oral glucose tolerance test. * Gestation confirmed by ultrasound. † Controls and decoys only. $\ddagger$ If referred for GDM management at any time (all intervention group subjects, and controls and decoys if OGTT indicates GDM at 24-28 weeks).

Missing values. Missing values will be analysed to determine whether they are random. In order to examine potential bias attributable to missing values, results derived from multiple imputed datasets will be compared with the results of the full analysis.

Sensitivity analysis. The results of a per protocol analysis will be compared with those of the intention-to-treat analysis.

Power calculations. With 400 women in each group, and assuming a $10 \%$ loss to follow-up, a $6 \%$ difference in primary pregnancy outcome and primary maternal outcome and a difference in primary neonatal outcome (lean body mass) of $74 \mathrm{~g}$ (expected overall mean, $2900 \mathrm{~g}$; standard deviation, $350 \mathrm{~g}$ ) can be detected. If $20 \%$ of women with risk factors ${ }^{6}$ have booking GDM, 4000 women need to be recruited to achieve 800 RCT participants (400 for booking GDM, treatment; 400 for booking GDM, no treatment).

Data management. A customised bespoke web-based database is linked with a data capture system using Redcap software (www. project-redcap.org).

\section{Ethics approval}

The study was approved by the South Western Sydney Local Health District Human Research Ethics Committee (reference, 15/LPOOL/551).

\section{Dissemination of results}

Data will be stored at the primary analysis site (Melbourne) and the study coordination site (Campbelltown). Results will be disseminated in peer-reviewed publications, at scientific meetings, through collaboration with research groups undertaking comparable studies, and in discussions with guideline authors and policy makers.

\section{Discussion}

This is the first large scale multi-centre RCT to assess whether women diagnosed with booking GDM before 20 weeks' gestation according to criteria for diagnosing hyperglycaemia at 24-28 weeks of pregnancy benefit from treatment for GDM. Further, 
5 Schedule for participants randomised to the non-active group

\begin{tabular}{|c|c|c|c|}
\hline Data collection & $\begin{array}{l}\text { Screening/ } \\
\text { booking }\end{array}$ & Prenatal & Post-birth \\
\hline Visit window & $\begin{array}{c}\text { Day of } \\
\text { recruitment } \\
<20 \text { weeks }\end{array}$ & $\begin{array}{l}24-28 \\
\text { weeks }\end{array}$ & $\begin{array}{c}\text { Before } \\
\text { discharge } \\
\text { (24-72 h) }\end{array}$ \\
\hline
\end{tabular}

Maternal procedures and measurements

Demographic data

$x$

Medical/obstetric history

(including GDM risk factors)*

Health/quality of life

questionnaire

Weight

Height

Blood pressure

OGTT booked

$\mathrm{HbA}_{\mathrm{lc}}$ level

Serum stored

$x$

$x$

$x$

$x$

$x$

$x$

$x$

$x$

Record of hospitalisations

Details of last ultrasound prior to birth

GDM/birth information

Postnatal OGTT (GDM

women only)

Adverse events

Length of stay (birth and

postnatal)

Neonatal procedures and measurements

Neonatal information

$x$

Neonatal outcomes

$x$

Information about feeding

$x$

NICU admission and length of

stay

$\mathrm{GDM}=$ gestational diabetes mellitus; $\mathrm{HbA}_{1 \mathrm{c}}=$ glycated haemoglobin;

$\mathrm{NICU}=$ neonatal intensive care unit; OGTT = oral glucose tolerance test. * Gestation confirmed by ultrasound. † If not previously collected.

we will analyse our findings to determine optimal diagnostic criteria for pregnant women before 20 weeks' gestation.

A key challenge was determining the most appropriate primary pregnancy outcome measure. We selected a composite outcome of hard endpoints similar to those of other major GDM RCTs. ${ }^{1,2,21} \mathrm{We}$ included significant macrosomia (birthweight $\geq 4.5 \mathrm{~kg}$ ) as part of this composite because, when predicted by ultrasonography, this birthweight is a recognised threshold for obstetric intervention. Lower thresholds for particular groups (eg, Asians) have not been published. The specific neonatal primary outcome of neonatal lean body mass captures potential harm resulting from fetal undernutrition caused by overtreatment of GDM, a risk factor for future cardio-metabolic disease. ${ }^{13}$ We also included the specific maternal primary outcome of pre-eclampsia. ${ }^{1,2}$ Neonatal hypoglycaemia is included as a secondary outcome because of concern about consistency in its measurement at different sites.

The ethics of withholding diagnosis and deferring treatment of the booking GDM control women was discussed by the investigators, as in some centres these women would be treated, potentially improving pregnancy outcomes. On the other hand, fetal harm caused by overtreatment is also possible. As there is no conclusive evidence for the benefit of treating women with slightly elevated glucose levels during early pregnancy, we do not believe withholding immediate treatment from such women to be unethical.

It is nevertheless important to minimise potential harm, and all participants will be fully informed about both potential benefits and harms. Safety measures include:

- Clinical staff may screen for GDM at any time at their discretion.

- Women with a fasting glucose level of $6.1-6.9 \mathrm{mmol} / \mathrm{L}$ or ODIP will be excluded from the trial.

- The OGTT is repeated at 24-28 weeks for all participants not already referred for GDM management.

- Clinical staff are made aware that a significant proportion of women not referred for early treatment (two-thirds) are decoys, and that their continued participation should not promote early intervention.

- After giving birth, all women who were diagnosed with GDM at any point during the study (including booking GDM control women with normal OGTT results at 24-28 weeks) will be advised of their OGTT results and the need for longer term follow-up, including an OGTT 6-12 weeks after delivery.

- Heel-prick glucose assessment for babies of all women in the RCT and decoy groups will be undertaken, including those of women diagnosed with booking GDM and normal OGTT results at $24-28$ weeks.

Most mothers are aware that collecting a screening heel-prick blood sample from all newborns is standard practice (for the Guthrie test). Similarly, mothers with GDM are aware of the importance of collecting a heel-prick blood sample to test for neonatal hypoglycaemia soon after birth, and opting out of this step will be an informed decision. However, participants not actively managed (decoys and most controls) will also provide consent for this extra heel-prick blood sample, as some women with booking GDM but normal OGTT results at 24-28 weeks might have fetuses at risk of neonatal hypoglycaemia. As a consequence of early GDM, fetal hyperinsulinaemia can normalise maternal glucose tolerance later in pregnancy through the fetal glucose steal phenomenon. $^{22,23}$

In a sub-analysis by OGTT strata (HRV $v$ LRV), we will prospectively investigate the benefits and harms of treating women with booking GDM. This analysis, together with an examination of the associations between booking glucose level, gestational age when commencing treatment, and adverse pregnancy outcomes across the whole cohort, should inform discussions about diagnostic thresholds if treatment is found to be beneficial. With continued follow-up of the chart-only and RCT control groups, it will be possible to undertake an epidemiological analysis of the association of booking glycaemia with adverse outcomes comparable with, but smaller than, the Hyperglycaemia and Adverse Outcomes (HAPO) study. ${ }^{24}$

In conclusion, TOBOGM is the first multi-centre RCT of the diagnosis and treatment of hyperglycaemia in pregnant women before 20 weeks' gestation according to diagnostic criteria for GDM at 24-28 weeks. We will explore the effects of treatment on pregnancy outcomes for women and their babies. The study will define the prevalence and natural history of booking GDM, examine treatment effects, and evaluate the cost-effectiveness of the screening, diagnosis and treatment of booking GDM. We expect that the results of the trial will guide future practice. 


\section{Author contributions}

David Simmons conceived the project, and drafted the initial trial design and this article. All co-authors contributed to the design of the study and the final manuscript. All authors have read and approved the final manuscript. David Simmons is guarantor.

\section{Funding statement}

The trial is funded by a grant from the National Health and Medical Research Council (APP1104231). Roche has provided meters for collecting blood glucose selfmonitoring data from women attending the diabetes-inpregnancy clinics.

Acknowledgements: We thank Charlene Thornton for her assistance with power calculations and initial statistical advice, Sanjeeva Ranasinha for the statistical analysis plan, Tony Keech for his advice on trial implementation, and Lisa Vizza, Jodie Nema and Claudia Bishop for assistance with the preparation of the trial for implementation.

Competing interests: No relevant disclosures.

(c) 2018 AMPCo Pty Ltd. Produced with Elsevier B.V. All rights reserved.
1 Crowther CA, Hiller JE, Moss JR, et al. Effect of treatment of gestational diabetes on pregnancy outcomes. $N$ Engl J Med 2005; 352: 2477-2486.

2 Landon MB, Spong CY, Thom E, et al. A multicenter, randomized trial of treatment for mild gestational diabetes. N Engl J Med 2009; 361: 1339-1348.

3 Nankervis A, McIntyre $H D$, Moses R, et al. ADIPS consensus guidelines for the testing and diagnosis of gestational diabetes mellitus in Australia and New Zealand. Nov 2014. http://adips.org/ downloads/2014ADIPSGDMGuidelinesV18.11.2014_000. pdf (viewed Jan 2017).

4 World Health Organization. Diagnostic criteria and classification of hyperglycaemia first detected in pregnancy. (WHO/NMH/MND/13.2). Geneva: WHO, 2013. http://apps.who.int/iris/bitstream/10665/85975/1/ WHO_NMH_MND_13.2_eng.pdf (viewed Jan 2017).

5 International Association of Diabetes and Pregnancy Study Groups Consensus Panel. International Association of Diabetes and Pregnancy Study Groups recommendations on the diagnosis and classification of hyperglycemia in pregnancy. Diabetes Care 2010; 33: 676-682.

6 Harreiter J, Simmons D, Desoye G, et al. IADPSG and WHO 2013 gestational diabetes mellitus criteria identify obese women with marked insulin resistance in early pregnancy. Diabetes Care 2016; 39: e90-e92.

7 Meyer WJ, Carbone J, Gauthier DW, Gottmann DA. Early gestational glucose screening and gestational diabetes. J Reprod Med 1996; 41: 675-679.

8 Super DM, Edelberg SC, Philipson EH, et al. Diagnosis of gestational diabetes in early pregnancy. Diabetes Care 1991; 14: 288-294.
9 Egan AM, Vellinga A, Harreiter J, et al. Epidemiology of gestational diabetes mellitus according to IADPSG/WHO 2013 criteria among obese pregnant women in Europe. Diabetologia 2017; 60: 1913-1921.

10 Langer O, Levy J, Brustman L, et al. Glycemic control in gestational diabetes mellitus: how tight is tight enough; small for gestational age versus large for gestational age? Am J Obstet Gynecol 1989; 161: 646-653.

11 Hattersley AT, Beards F, Ballantyne E, et al. Mutations in the glucokinase gene of the fetus result in reduced birth weight. Nat Genet 1998; 19: 268-270.

12 Catalano PM, Mele L, Landon MB, et al; Eunice Kennedy Shriver National Institute of Child Health and Human Development Maternal-Fetal Medicine Units Network. Inadequate weight gain in overweight and obese pregnant women: what is the effect on fetal growth? Am J Obstet Gynecol 2014; 211: el-el37.

13 Hales CN, Barker DJ. Type 2 (non-insulin-dependent) diabetes mellitus: the thrifty phenotype hypothesis. Diabetologia 1992; 35: 595-601.

14 Mills JL, Jovanovic L, Knopp R, et al. Physiological reduction in fasting plasma glucose concentration in the first trimester of normal pregnancy: the Diabetes in Early Pregnancy Study. Metabolism 1998; 47: $1140-1144$.

15 Zhu WW, Yang HX, Wei YM, et al. Evaluation of the value of fasting plasma glucose in the first prenatal visit to diagnose gestational diabetes mellitus in China. Diabetes Care 2013; 36: 586-590.

16 McIntyre HD, Sacks DA, Barbour LA, et al. Issues with the diagnosis and classification of hyperglycemia in early pregnancy. Diabetes Care 2016; 39: 53-54.
17 Morrison MK, Collins CE, Lowe JM. Dietetic practice in the management of gestational diabetes mellitus: a survey of Australian dietitians. Nutr Diet 2011; 68: 189-194.

18 Sacks, DB, Arnold M, Bakris GL, et al. Guidelines and recommendations for laboratory analysis in the diagnosis and management of diabetes mellitus. Diabetes Care 2011; 34: e61-e99.

19 International Association of Diabetes in Pregnancy Study Group (IADPSG) Working Group on Outcome Definitions; Feig DS, Corcoy R, Jensen DM, et al. Diabetes in pregnancy outcomes: a systematic review and proposed codification of definitions. Diabetes Metab Res Rev 2015 31: 680-690.

20 Lowe SA, Bowyer L, Lust K, et al. SOMANZ guidelines for the management of hypertensive disorders of pregnancy. Aust NZ Obstet Gynaecol 2014; 55: el-e29.

21 Rowan JA, Hague WM, Gao W et al. Metformin versus insulin for the treatment of gestational diabetes. $N$ Engl J Med 2008; 358: 2003-2015.

22 Desoye G, Nolan CJ. The fetal glucose steal: an underappreciated phenomenon in diabetic pregnancy. Diabetologia 2016; 59: 1089-1094.

23 Sovio U, Murphy HR, Smith GC. Accelerated fetal growth prior to diagnosis of gestational diabetes mellitus: a prospective cohort study of nulliparous women. Diabetes Care 2016 39: 982-987.

24 HAPO Study Cooperative Research Group; Metzger BE, Lowe LP. Dyer AR, et al. Hyperglycemia and adverse pregnancy outcomes. N Engl J Med 2008; 358: 1991-2002. 